\title{
Lrig1 Expression in Human Sebaceous Gland Tumors
}

\author{
Jöri Pünchera Laurent Barnes Gürkan Kaya \\ Department of Dermatology, University Hospital of Geneva, Geneva, Switzerland
}

\section{Key Words}

Sebaceous gland · Lrig1 · CD44 · Stem cells · Sebaceous tumor · Sebaceous carcinoma ·

Sebaceous adenoma

\begin{abstract}
Background: Sebaceous glands contribute significantly to the barrier functions of the skin. However, little is known about their homeostasis and tumorigenesis. Recently, increased expression of stem cell marker Lrig1 has been reported in sebaceous carcinoma-like tumors of $\mathrm{K} 14 \Delta$ NLef1 transgenic mice. In this study, we analyzed the Lrig1 expression in human sebaceous tumors. Methods: Twenty-eight formalin-fixed paraffin-embedded sebaceous tumor specimens (7 sebaceous hyperplasias, 7 sebaceous adenomas, 10 sebaceomas and 4 sebaceous carcinomas) were stained with anti-Lrig1, anti-CD44v3 and anti-Ki67 antibody. Results: Four (100\%) sebaceous carcinomas, 8 (80\%) sebaceomas, 3 (43\%) sebaceous adenomas and no sebaceous hyperplasia showed Lrig1 overexpression. Discussion and Conclusion: Lrig1 is a known tumor suppressor gene and is usually considered to be an indicator of poorly aggressive tumors. In human sebaceous tumors, the stronger Lrig1 staining in sebaceous carcinoma compared to other sebaceous tumors might be a feature of an advanced stage in tumorigenesis and a bad prognosis. In our study, $100 \%$ of sebaceous carcinomas revealed Lrig1 overexpression. We propose that Lrig1 may be used as a possible new marker of poorly differentiated sebaceous carcinoma.

\section{Introduction}

The sebaceous glands cover the whole body surface except the palms of hands and the soles of feet. They are believed to contribute to the antibacterial properties of the skin [1-6], the hydration of the skin surface [7] and the cutaneous vitamin E and other antioxidant 
compound synthesis [8], and to produce part of the lipids of the stratum corneum [9]. They do so by excreting to the skin surface an oily waxy substance called sebum, the end product of the holocrine secretion of sebocytes [10]. Mutations that abrogate the lipid synthesis have shown to induce serious disruption of the epidermal barrier function in mouse [9]. In humans, defects of the sebaceous gland function cause several diseases from acne $[11,12]$ to sebaceous carcinoma (SC). Malignant transformation of the sebaceous gland leads to SC, an aggressive tumor [13] that is often diagnosed late and carries a high mortality rate [14]. The sebaceous gland has only recently been recognized to be of crucial importance for the maintenance of healthy skin. That is why several recent studies focused on the understanding of the sebaceous gland homeostasis and its contribution to the whole epidermal homeostasis.

Different niches of stem cells, which individually contribute to the homeostasis of an epidermal compartment, have been described in mice [15-17]. The interfollicular epidermis (IFE) was shown to be regenerated according to an autocrine $\mathrm{Wnt} / \beta$-catenin pathway, with each cell of the basal layer being able to divide according an asymmetric model of division $[18,19]$. But Lrig1+ keratinocytes from the isthmus, located in the hair follicle at the junctional zone between the sebaceous gland, hair follicle and IFE, were shown to be able to renew the whole epidermis in case of injury. Lrig1+ cells replenish the infundibulum, the isthmus and the sebaceous gland in steady-state conditions, and can upon injury replenish the whole epidermis including the IFE [20]. That is why Lrig1 cells are considered to be epidermal multipotent stem cells. Lgr6+ cells, which are located above the bulge of the hair follicle, are also able to renew all the compartments of the epidermis [21]. In human epidermis, the Lrig1 niche is composed of Lrig1+ clusters of keratinocytes in the basal layer. These clusters were described to be on the top of the rete ridges of the IFE [22]. More recently, we have shown a second niche of Lrig1+ keratinocytes, more similar to the mouse niche as located in the human isthmus connecting the hair follicle with the sebaceous gland [23]. During the morphogenesis of the folliculosebaceous unit (FSU), divided into 8 stages [24, 25], the Lrig1+ cells are considered to be sebocyte precursor cells [26]. Sox9 represents a well-known hair follicle stem cell marker [27, 28], which is also indispensable for the morphogenesis of the FSU [28]. At the beginning of the morphogenesis, the Lrig1+ cells are superposed with the Sox9+ cells, but at stage 5 of morphogenesis, the Lrig1+ cells dissociate from the Sox9+ cells [26]. The Sox $9+$ cells stay at the bulge, to form the hair follicle, whereas the Lrig1+ cells migrate more distally to the isthmus. At the isthmus the first sebocyte will appear after asymmetric cell division [26]. The first sebocytes, which contain an essential enzyme for the triglyceride synthesis called stearoyl-CoA desaturase 1 (SCD1) [29, 30], appear at stage 5 of morphogenesis. These SCD1+ sebocytes are themselves Lrig1- but surrounded by Lrig1+ precursor cells [26].

Lrig1 is a transmembrane protein whose biological function is to repress the epidermal growth factor receptor (EGFR) [31]. Secreted growth factors can bind to EGFR and promote epidermal growth [32]. Therefore, Lrig1 is expected to act as an inhibitor of tissue growth that maintains the cells in a quiescent state. Logically, downregulation of Lrig1 has been associated with diseases showing epidermal hyperplasia like psoriasis. Lrig1 mutant mice also showed a psoriasis-like phenotype [33]. Recent studies showed that EPGN (epithelial mitogen) supply (EPGN is a specific ligand of EGFR) to an embryo before stage E11.5, in other words well before the epidermal stratification or the pilosebaceous morphogenesis, leads, in an EGFR dependent manner, to postnatal hypertrophy of the sebaceous glands. This hypertrophy is maintained as long as EPGN supply is high and the glands diminish in size after withdrawal of EPGN. It is interesting to mention that these hypertrophic sebaceous glands have a strong Lrig1 staining in the sebocytes. In addition, these glands logically showed an increase in c-myc expression, as this one is a regulator of epidermal growth downstream of the EGFR pathway [34]. 
A murine model of sebaceous gland tumors has recently been described. There, a dominant negative form of Lef1 has been expressed specifically in the epidermis of transgenic mice. Upon treatment with a mutagenic agent, all mice developed tumors of the sebaceous glands, indicating that mutations in the Wnt/ $\beta$-catenin pathway are crucial for the malignant transformation of keratinocytes in sebaceous carcinoma-like tumor (SCLT). K14 $\Delta$ NLef1 mice had a mutation of the $\mathrm{NH}_{2}$-terminus of Lef1 expressed under the control of keratin 14 promoter which hinders $\beta$-catenin from binding to Lef1. These mice develop spontaneous sebaceous adenomas (SA) in a normal genetic background. However, under constitutive activation of Rac1, these adenomas evolve to poorly differentiated SCLT. Lrig1 is highly expressed in these poorly differentiated SCLTs compared to SA [35].

In this study, we explored the expression of Lrig1 in human sebaceous tumors. SCLTs induced in a murine model expressing a dominant active form of Lef1 specifically in the basal layer (K14 $\Delta$ NLef1) were shown to be all positive for Lrig1. We then analyzed human sebaceous tumors for their Lrig1-expressing status in order to confirm this murine model. Since a variant of CD44, CD44v3, has recently been shown to be present in the human FSU together with Lrig1 [23], we also explored its expression in sebaceous tumors.

\section{Methods}

We searched the biopsy database of the Dermatopathology Unit of the Department of Dermatology/University Hospital of Geneva for sebaceous tumors from 2004 to 2015; 8 SC, 35 SA, 2 sebaceous epitheliomas, 13 sebaceomas (Se) and 35 sebaceous hyperplasias (SH) were found. According to the new nomenclature, proposed by Troy and Ackerman in 1984, we added sebaceous epithelioma into the Se group [36]. We visualized all the sebaceous tumors on bright field microscopy. For this study, we then selected the ones with the clearest or the least ambiguous diagnosis on HE staining: 4 SC, 7 SA, 10 Se and $7 \mathrm{SH}$.

All selected biopsy samples were formalin fixed and paraffin embedded; 5 - $\mu$ m-thin slices were cut, fixed on Superfrost Ultraplus object slides and dried overnight at room temperature. After the deparaffinization by using a robot, the slides were put in a boiling citrate buffer (10 mM, pH 6) for $15 \mathrm{~min}$ and cooled down at room temperature for $1 \mathrm{~h}$. Three baths in PBS (phosphate buffered saline) $+0.3 \%$ TritonX-100-T, 5 min each, followed. The slides were then saturated with a 15-min bath in PBS $+0.3 \%$ TritonX-100 + 1\% BSA (bovine serum albumin) $+1 \%$ milk powder for 15 min before incubation with the first antibody overnight at $4{ }^{\circ} \mathrm{C}$, diluted in PBS $+0.3 \%$ TritonX-100 $+1 \% \mathrm{BSA}+2 \%$ milk powder. The following antibodies with the following dilutions were used: rabbit anti-human Lrig1 antibody diluted 1/5,000 $[22,37,38]$ provided by Satoshi Itami, Osaka University, mouse anti-human CD44v3 antibody diluted 1/100 (Bender MedSystems, BMS144) and monoclonal mouse anti-human Ki67 antibody diluted 1/100 (Dako, M7240).

The next day, the slides were warmed up at room temperature and rinsed in TBS-T (Tris $0.1 \mathrm{M}, \mathrm{NaCl} 0.9 \%$, Tween $0.3 \%, \mathrm{pH} 7.6) 3$ times for $5 \mathrm{~min}$. Then, there were two different protocols: immunofluorescence and immunohistochemistry.

\section{Immunofluorescence}

The secondary antibody (dilution $1 / 200$ ) plus DAPI (dilution $1 / 200$ ) for DNA counterstaining were added, all diluted in TBS-T $+1 \%$ BSA $+2 \%$ milk powder. For the secondary antibody, either an anti-mouse or an anti-rabbit antibody, depending on the host in which the first antibody was produced, was used. After an incubation for $60 \mathrm{~min}$, the slides were rinsed again in TBS-T 3 times for $5 \mathrm{~min}$ and $5 \mathrm{~min}$ in PBS before mounting the object with a fluorescence mounting medium (Dako, S3023). 


\section{Immunohistochemistry}

The slides incubated with the Ki67 antibody were analyzed on immunohistochemistry and revealed with DAB. For this series, the Vectastain Elite ABC Kit (Vector Laboratories, PK-6200) was used according to a standard protocol. The slides were counterstained with a solution made of 1 tablet DAB (Sigma DAB, D5905) dissolved in $15 \mathrm{ml}$ Tris-buffered saline, $\mathrm{pH}$ 7.6, for $150 \mathrm{~s}$ before stopping the reaction in a distilled water bath. Then, the object was mounted.

To visualize the results and capture the pictures, a Pannoramic digital slide scanner 250 (3DHISTECH) and a microscope confocal system Leica SP5 were used. We calculated the degree of over- or underexpression, by scoring the staining in sebaceous tumors from 0 to 4 . Where 0 means absent staining, 1 means normal expression as expected in normal sebaceous glands, 2 indicates moderate and 3 strong staining respectively. The highest score is 4, meaning very strong Lrig1 staining. It is worth mentioning that for the Ki67 staining, absent staining (as would be expected in normal sebaceous glands) was scored 1. Thus, in every sebaceous tumor sample, a value above 1 signifies higher expression and a value below 1 lower expression of the respective marker compared to normal sebaceous glands. We then compared the marker expression between different sebaceous gland tumors by calculating the respective marker's mean values. To judge statistical significance, we calculated the $p$ value according to the Student's t test.

\section{Results}

We first analyzed Lrig1 expression in normal human skin. We confirmed the already known Lrig1+ niche in the basal cells of human IFE and found a second Lrig1 niche at the isthmus of human sebaceous glands [23]. These results are in concordance with the known Lrig1+ niche in murine epidermis [20,22]. We then analyzed 28 samples of human sebaceous tumors for the expression of Lrig1, CD44v3 and Ki67. Table 1 and figure 1 show an overview of the obtained results. A comparison of the mean values in different sebaceous tumors is presented in table 2 .

All SC showed Lrig1 overexpression, compared to $43 \%$ of SA, $80 \%$ of Se and $0 \%$ of SH. SC showed the highest mean Lrig1 score (3.25), followed by Se (2.3), SA (2.0) and SH (1.0). The difference in the Lrig1 score between SC and SH was statistically significant ( $p=0.0001$; table 3). We found an inverse relationship between Lrig1 overexpression and the degree of differentiation. SC also presented the highest Ki67 score with 3, compared to 2.143 for SA and 2 for Se. The difference in Ki67 score between SC and other sebaceous tumors was statistically significant (table 3). In SC, we observed a slight CD44v3 downregulation scored at 0.75 . SA had a mean CD $44 \mathrm{v} 3$ score of 0.857 , Se 1.2 and SH 1 . None of the differences in the CD44v3 score between SC and other sebaceous tumors was statistically significant. CD44v3 staining was absent in $67 \%$ of samples with very strong Lrig1 overexpression.

\section{Discussion and Conclusion}

Lrig1 and CD44 have both been widely discussed in cancer prognosis. The data of Lrig1 expression in tumors tend to define it as a good prognostic factor, in agreement with its tumor suppressor function [39]. Lrig1 ablation in mice induced duodenal cancers, which enforces the hypothesis that Lrig1 is a tumor suppressor gene [40]. High Lrig1 expression is an indicator of good prognosis in squamous cell carcinoma of the skin [37], in estrogen receptor- $\alpha$ breast cancer [41] and in non-small cell lung cancer $[42,43]$. On the other hand, loss of Lrig1 
Table 1. Summary of Lrig1, CD44v3 and Ki67 staining in human sebaceous tumors
Table 2. Comparison of mean values of Lrig1, CD44v3 and Ki67 staining
(C) 2016 The Author(s). Published by S. Karger AG, Basel www.karger.com/dpa

Pünchera et al.: Lrig1 Expression in Human Sebaceous Gland Tumors

\begin{tabular}{lllll}
\hline Case & Lrig1 & CD44v3 & Ki67 & Differentiation \\
\hline SH 1 & 1 & 1 & 2 & $\mathrm{~W}$ \\
SH 2 & 1 & 1 & 2 & $\mathrm{~W}$ \\
SH 3 & 1 & 1 & 2 & $\mathrm{~W}$ \\
SH 4 & 1 & 1 & 1 & $\mathrm{~W}$ \\
SH 5 & 1 & 1 & 1 & $\mathrm{~W}$ \\
SH 6 & 1 & 1 & 1 & $\mathrm{~W}$ \\
SH 7 & 1 & 1 & 1 & $\mathrm{~W}$ \\
SA 1 & 4 & 0 & 2 & $\mathrm{M}$ \\
SA 2 & 1 & 1 & 2 & $\mathrm{M}$ \\
SA 3 & 0 & 0 & 2 & $\mathrm{P}$ \\
SA 4 & 4 & 1 & 3 & $\mathrm{M}$ \\
SA 5 & 1 & 1 & 2 & $\mathrm{M} / \mathrm{P}$ \\
SA 6 & 3 & 2 & 2 & $\mathrm{~W}$ \\
SA 7 & 1 & 1 & 2 & $\mathrm{~W}$ \\
Se 1 & 4 & 1 & 2 & $\mathrm{M} / \mathrm{P}$ \\
Se 2 & 2 & 2 & 2 & $\mathrm{M} / \mathrm{P}$ \\
Se 3 & 4 & 0 & 2 & $\mathrm{M} / \mathrm{P}$ \\
Se 4 & 2 & 1 & 2 & $\mathrm{M}$ \\
Se 5 & 3 & 1 & 2 & $\mathrm{M} / \mathrm{W}$ \\
Se 6 & 2 & 2 & 2 & $\mathrm{M}$ \\
Se 7 & 3 & 3 & 2 & $\mathrm{M} / \mathrm{W}$ \\
Se 8 & 0 & 0 & 2 & $\mathrm{M} / \mathrm{P}$ \\
Se 9 & 3 & 1 & 2 & $\mathrm{P}$ \\
Se 10 & 0 & 1 & 2 & $\mathrm{M}$ \\
SC 1 & 4 & 0 & 3 & $\mathrm{P}$ \\
SC 2 & 3 & 1 & 3 & $\mathrm{M}$ \\
SC 3 & 2 & 2 & 2 & $\mathrm{P}$ \\
SC 4 & 4 & 0 & 4 & $\mathrm{P}$ \\
\hline
\end{tabular}

$\mathrm{SG}=$ Sebaceous gland; $\mathrm{W}=$ well differentiated; $\mathrm{M}=$ moderately differentiated; $\mathrm{P}=$ poorly differentiated; $0=$ absent staining $(-) ; 1=$ normal staining as expected in normal sebaceous glands $(+) ; 2=$ moderate staining $(++) ; 3=$ strong staining $(+++) ; 4$ = very strong staining $(++++)$.

\begin{tabular}{llllll}
\hline & SH & SA & Se & SC & Overall \\
\hline Lrig1 & 1 & 2 & 2.3 & 3.25 & 2.036 \\
CD44v3 & 1 & 0.857 & 1.2 & 0.75 & 1 \\
Ki67 & 1.429 & 2.143 & 2 & 3 & 2.036 \\
\hline
\end{tabular}

Values $>1$ indicate higher expression; values $<1$ indicate lower expression of the respective marker compared to normal sebaceous glands.

or low Lrig1 expression is hypothesized to be a predictor of poor survival and a risk factor for metastasis in breast cancer patients [44]. In contrast, CD44 would rather be a marker of sebocyte differentiation, while Lrig1 is a marker of sebocyte stem cells in mice and in humans [23]. Additionally, high expression of CD44 has been associated with bad prognosis and high metastatic capacity in lung cancer [45]. CD44 is thought to enhance several pro-oncogenic signals $[45,46]$. A meta-analysis showed that CD44 expression has possibly negative effects 


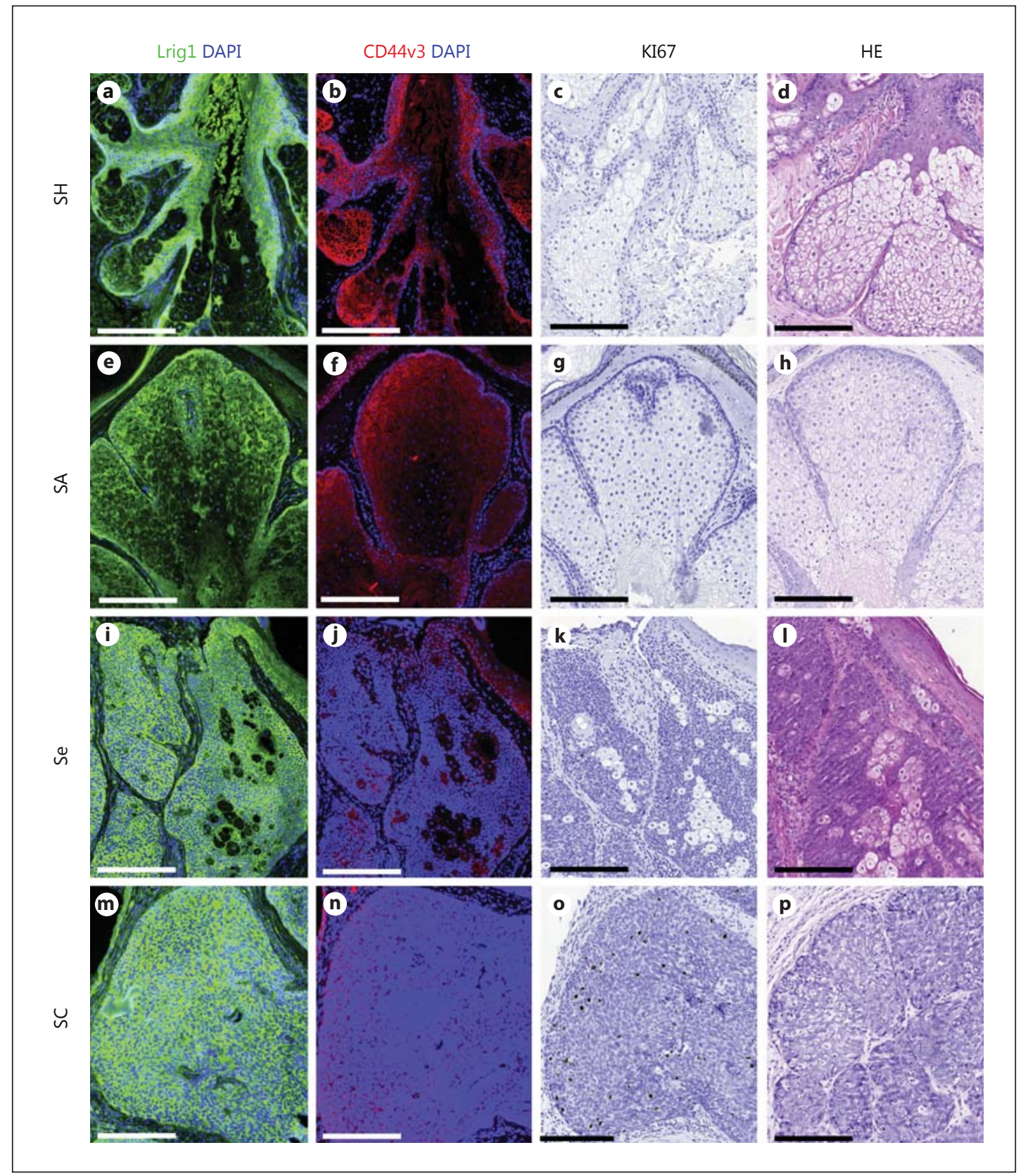

Fig. 1. Lrig1, CD44v3 and Ki67 staining in human sebaceous tumors. Serial slides of SH (a-d), SA (e-h), Se $(\mathbf{i}-\mathbf{I})$ and SC (m-p); Lrig1 is in green (immunostaining), CD44v3 in red (immunostaining), Ki67 in black (immunostaining) and DAPI in blue (indicates DNA). Bar $=200 \mu \mathrm{m}$.

on survival in gastric cancer [47]. CD44 overexpression was associated with a poor prognostic outcome in non-small cell lung cancer [48]. At the same time, loss of CD44 correlates also with poorer clinical outcome in gastric stromal tumors [49]. However, in the SC of the eyelid, CD44 expression offers no prognostic value [50].

As described above, since Lrig1 is considered to be a tumor suppressor gene, an increased Lrig1 staining is usually an indicator of good prognosis. Concerning sebaceous tumors, our results cannot definitively state whether Lrig1 expression is a marker of malignant SC because 
Table 3. $p$ values of differences in marker staining
(C) 2016 The Author(s). Published by S. Karger AG, Base www.karger.com/dpa

Pünchera et al.: Lrig1 Expression in Human Sebaceous Gland Tumors

\begin{tabular}{lllll}
\hline & SC vs. SH & SC vs. SA & SC vs. Se & SC vs. overall \\
\hline Lrig1 & 0.0001 & 0.2 & 0.247 & 0.054 \\
CD44v3 & 0.489 & 0.833 & 0.429 & 0.464 \\
Ki67 & 0.004 & 0.038 & 0.001 & 0.0003 \\
\hline
\end{tabular}

$\mathrm{p}$ values (calculated according to Student's t test) of the difference in intensity of marker staining between different sebaceous tumors.

of a lack of statistical power. However, all of the 4 SCs analyzed were positive for Lrig1, and SC had a higher Lrig1 score than other sebaceous tumors. Although, this higher Lrig1 score of $\mathrm{SC}$ compared to other sebaceous tumors is statistically significant only if the Lrig1 score is compared between SC and SH (table 3). This observation then tends to confirm the animal model reported by Frances et al. [35]. In this study, activation of Rac1 was sufficient to transform SA into malignant SC in K14 $\Delta$ NLef1 mutant mice presenting spontaneous SA. The $\mathrm{SC}$ that developed in these mice were shown to lose the sebaceous differentiation and to express high levels of the Lrig1 stem cell marker. That is actually a feature we have found in the $4 \mathrm{SC}$ analyzed here, again confirming the data from the mouse model reported by Frances et al. [35]. Additionally, Ki67 (marker of proliferation) was also high in our 4 SC human samples, when compared with SA or Se, confirming the higher aggressivity of the SC. Among the 4 cases of SC, only one presented a poor signal for CD44v3 staining, while the other 3 were negative. If CD44v3 is then considered as a sebaceous differentiation marker, this result then confirms that SCs are poorly differentiated tumors, as already indicated by their high expression level of Lrig1 and in agreement with the murine model described by Frances et al. [35].

Regarding the 7 cases of SA, cases of less aggressive sebaceous tumors, 3 showed Lrig1 overexpression, whereas 4 did not. Then, globally, the Lrig1 signal appeared to be less intense in SA (mean Lrig1 score of 2 compared to 3.25 in SC). The p value for this difference in Lrig1 score was at 0.2 and thus not statistically significant, possibly due to the low number of SC. They also tend to present a higher degree of differentiation. This is also in agreement with the animal model reported by Frances et al. [35]. However, Lrig1 overexpression may not be a marker to very strictly differentiate SC from SA from a histopathological point of view. Indeed, some SA may show Lrig1 overexpression and some may not, while all SC show Lrig1 overexpression. However, the absence of Lrig1 overexpression may help discriminate SA from SC. Whether Lrig1 overexpression is a good survival prognostic marker cannot be answered by this study as the survival rates of the patients included in this study have not been analyzed. Nevertheless, these are interesting data that can be used to evaluate Lrig1 overexpression in SA and to make a potential correlation with higher chances of transformation into SC.

Similar comments can be made for the Lrig1 staining performed on the 10 cases of Se in this study. Se showed a slightly higher mean Lrig1 score than SA: 2.3 in Se compared to 2.0 in SA. Again, this difference is not statistically significant. However, in Se, 8 out of 10 (80\%) cases showed some degree of Lrig1 overexpression. This higher rate of Lrig1 overexpression in Se, compared to only $43 \%$ (3 out of 7 cases) in SA, may confirm that Se is a type of lesion between SA and SC, and thus more likely to transform into SC than SA.

Concerning SH, which is a benign lesion, none of the 7 cases analyzed showed Lrig1 overexpression. SH had an Lrig1 score of 1.0. The higher Lrig1 score in SC compared to SH was statistically significant (table 3). Additionally, SH had a statistically significant lower Lrig1 score than Se (data not shown). This finding could confirm that poor Lrig1 expression is a sign 
of a benign lesion in sebaceous tumors. SC, SA and Se can potentially be part of the Muir-Torre syndrome (a rare genodermatosis that presents itself by sebaceous neoplasms associated with at least one visceral malignancy) and can therefore all have aggressive behavior [51]. All of these three sebaceous tumors had a mean Lrig1 score of $\geq 2$, and the mean Lrig1 score in $\mathrm{SH}$ was 1 . In our study, although limited by a small number of tumor samples, the most aggressive sebaceous tumor (SC) showed the strongest Lrig1 staining, and the most benign pathology (SH) showed no increase in Lrig1 staining compared to normal sebaceous glands. Therefore, in sebaceous tumors, Lrig1 overexpression might be the sign of an advanced stage of tumorigenesis.

Recently, a study showed a murine model where deletion of Ptch1 leads to the development of basal cell carcinoma (BCC)-like tumors [52]. These tumors derive from stem cells situated at the upper/lower bulge and the isthmus. Interestingly, all these BCC-like tumors are Lrig1+ [52], similar to the SCLT reported by Frances et al. [35]. Our study confirms Lrig1 positivity in all analyzed human SC. Peterson et al. [52] reported that BCC preferentially originate from epidermal stem cells, among which they identified Lrig1+ cells located in the murine isthmus as inducers of BCC-like tumors. Since we have demonstrated earlier that cells located in the human isthmus also express Lrig1 [23], the positivity of Lrig1 in all human SC found in this study may suggest that human SC also derive from Lrig1+ cells originally located in the isthmus at the junction between the sebaceous gland and hair follicle.

As explained above, a relationship between CD44 and poor survival has been hypothesized in various cancers. Considering the general pro-oncogenic behavior of CD44-positive cells, we would expect an overexpression of CD44 in the most aggressive sebaceous tumors. Unfortunately, we cannot confirm this finding in our study. The most malignant sebaceous tumor (SC) was only seen in 1 out of $4(25 \%)$ cases; CD44v3 overexpression was observed in $14.3 \%$ in SA and 30\% in Se. SH never showed CD44v3 overexpression. Indeed, a downregulation of CD44v3 was found in the most aggressive tumor, as SC showed a CD44v3 score of 0.75 . This could confirm on one hand the poor grade of differentiation of SC, as described in murine SC by Frances et al. [35], and on the other hand this might be explained by the strong Lrig1 signal which dominates in sebaceous tumors, the biological activity of which was shown to repress differentiation. This is also in line with the exclusion of CD44v3 expression from the Lrig1+ clusters identified in IFE [53]. This inverse relationship of Lrig1 and CD44v3 can be observed as no CD44v3 overexpression is noticed in $67 \%$ of sebaceous tumor samples with some degree of Lrig1 overexpression. Of the sebaceous tumor samples with very strong Lrig1 overexpression, CD44v3 staining was absent in two thirds of cases. Overall, CD44v3 staining showed no significant difference in different sebaceous tumors. Therefore, CD44v3 expression does not seem to be an indicator of tumor aggressivity in sebaceous gland tumors.

Ki67 is a known marker of proliferation and is expected to be overexpressed in highly proliferative cells such as sebaceous tumor cells. As expected, the most aggressive tumor (SC) has the highest mean Ki67 score. This higher Ki67 score in SC compared to other sebaceous tumors is statistically significant (table 3). The higher the Ki67 staining, the more poorly differentiated the sebaceous tumor samples. $100 \%$ of the samples with absent Ki67 staining are well differentiated, compared to only $25 \%$ of samples with moderate Ki67 and $0 \%$ of samples with frank Ki67 staining. This confirms the findings in human sebaceous tumor samples of Cottle et al. [54] and the findings in the murine model of sebaceous tumors reported by Frances et al. [35].

As we go from SH to SA, Se and finally SC, the degree of differentiation is expected to decline with the rise of uncontrolled proliferation of tumor cells in more aggressive tumors. Our study confirms this, as $75 \%$ of SC was poorly differentiated, compared to $14.3 \%$ of SA, $10 \%$ of Se and $0 \%$ of SH. On the other hand, $0 \%$ of SC and Se are well differentiated, compared to $28.6 \%$ of SA and $100 \%$ of SH. Interestingly, $70 \%$ of poorly or moderately to poorly differ- 
entiated sebaceous tumor samples had some degree of Lrig1 overexpression. Of all the samples with Lrig1 overexpression, only $6.67 \%$ were well differentiated. This might be an Lrig1-independent observation. Since in sebaceous tumors Lrig1 expression rises proportionally to the increase in malignancy, the samples with the poorest differentiation happen to have the strongest Lrig1 expression.

We confirm the findings of Ki67 overexpression and poor sebocyte differentiation in human SC [54]. CD44v3 staining tends to be slightly lower in SC and shows an inverse relationship with Lrig1 staining. Further studies are needed to explain the relationship of Lrig1 and CD44v3 in human sebaceous tumorigenesis and homeostasis. We conclude that human SC has an Lrig1 overexpression. SC has a stronger Lrig1 staining than SH and possibly as SA and Se. We therefore confirm the findings of the previously reported murine model of sebaceous tumors [35] which are also applicable in human sebaceous tumors. The deeper the sebocytes enter tumorigenesis, the stronger the Lrig1 staining. We propose that Lrig1 may be used as a possible new marker for poorly differentiated SC.

\section{Acknowledgments}

This work was supported by the Fondation Eugène et Georges Gelbert, Geneva, Switzerland. We are grateful to Dr. Satoshi Itami (Osaka University, Japan) for kindly providing us with the Lrig1 antibody.

\section{Statement of Ethics}

Skin samples, previously collected for medical diagnostic, were obtained from the histopathology collection of Dermatopathology Unit of the University Hospital of Geneva. They were treated anonymously.

\section{Disclosure Statement}

The authors declare no conflict of interests.

\section{References}

1 Drake DR, Brogden KA, Dawson DV, Wertz PW: Thematic review series: skin lipids. Antimicrobial lipids at the skin surface. J Lipid Res 2008;49:4-11.

2 Fischer CL, Drake DR, Dawson DV, Blanchette DR, Brogden KA, Wertz PW: Antibacterial activity of sphingoid bases and fatty acids against Gram-positive and Gram-negative bacteria. Antimicrob Agents Chemother 2012; $56: 1157-1161$.

3 Fischer CL, Blanchette DR, Brogden KA, et al: The roles of cutaneous lipids in host defense. Biochim Biophys Acta Mol Cell Biol Lipids 2014;1841:319-322.

4 Brogden NK, Mehalick L, Fischer CL, Wertz PW, Brogden KA: The emerging role of peptides and lipids as antimicrobial epidermal barriers and modulators of local inflammation. Skin Pharmacol Physiol 2012;25:167181.

5 Feingold KR: The outer frontier: the importance of lipid metabolism in the skin. J Lipid Res 2009; 50(suppl):S417-S422.

6 Georgel P, Crozat K, Lauth X, et al: A toll-like receptor 2-responsive lipid effector pathway protects mammals against skin infections with gram-positive bacteria. Infect Immun 2005;73:4512-4521.

7 Fluhr JW, Mao-Qiang M, Brown BE, et al: Glycerol regulates stratum corneum hydration in sebaceous gland deficient (asebia) mice. J Invest Dermatol 2003;120:728-737. 
8 Thiele JJ, Weber SU, Packer L: Sebaceous gland secretion is a major physiologic route of vitamin E delivery to skin. J Invest Dermatol 1999;113:1006-1010.

9 Binczek E, Jenke B, Holz B, Gunter RH, Thevis M, Stoffel W: Obesity resistance of the stearoyl-CoA desaturasedeficient $\left(\mathrm{scd}^{-/-}\right)$mouse results from disruption of the epidermal lipid barrier and adaptive thermoregulation. Biol Chem 2007;388:405-418.

10 Thody AJ, Shuster S: Control and function of sebaceous glands. Physiol Rev 1989;69:383-416.

11 Zouboulis CC, Jourdan E, Picardo M: Acne is an inflammatory disease and alterations of sebum composition initiate acne lesions. J Eur Acad Dermatol Venereol 2014;28:527-532.

12 Downie MM, Guy R, Kealey T: Advances in sebaceous gland research: potential new approaches to acne management. Int J Cosmet Sci. 2004;26:291-311.

13 Margo CE, Mulla ZD: Malignant tumors of the eyelid: a population-based study of non-basal cell and nonsquamous cell malignant neoplasms. Arch Ophthalmol 1998;116:195-198.

14 Buitrago W, Joseph AK: Sebaceous carcinoma: the great masquerader: emerging concepts in diagnosis and treatment. Dermatol Ther 2008;21:459-466.

15 Blanpain C, Fuchs E: Epidermal homeostasis: a balancing act of stem cells in the skin. Nat Rev Mol Cell Biol 2009;10:207-217.

16 Ghazizadeh S, Taichman LB: Multiple classes of stem cells in cutaneous epithelium: a lineage analysis of adult mouse skin. EMBO J 2001;20:1215-1222.

17 Niemann C, Horsley V: Development and homeostasis of the sebaceous gland. Semin Cell Dev Biol 2012;23: 928-936.

18 Page ME, Lombard P, Ng F, Gottgens B, Jensen KB: The epidermis comprises autonomous compartments maintained by distinct stem cell populations. Cell Stem Cell 2013;13:471-482.

19 Clayton E, Doupe DP, Klein AM, Winton DJ, Simons BD, Jones PH: A single type of progenitor cell maintains normal epidermis. Nature 2007;446:185-189.

20 Jensen KB, Collins CA, Nascimento E, et al: Lrig1 expression defines a distinct multipotent stem cell population in mammalian epidermis. Cell Stem Cell 2009;4:427-439.

21 Snippert HJ, Haegebarth A, Kasper M, et al: Lgr6 marks stem cells in the hair follicle that generate all cell lineages of the skin. Science 2010;327:1385-1389.

22 Jensen KB, Watt FM: Single-cell expression profiling of human epidermal stem and transit-amplifying cells: Lrig1 is a regulator of stem cell quiescence. Proc Natl Acad Sci USA 2006;103:11958-11963.

23 Barnes L, Puenchera J, Saurat JH, Kaya G: Lrig1 and CD44v3 Expression in the human folliculosebaceous unit. Dermatology 2015;231:116-118.

24 Paus R, Muller-Rover S, Van Der Veen C, et al: A comprehensive guide for the recognition and classification of distinct stages of hair follicle morphogenesis. J Invest Dermatol 1999;113:523-532.

25 Millar SE: Molecular mechanisms regulating hair follicle development. J Invest Dermatol 2002;118:216-225.

26 Frances D, Niemann C: Stem cell dynamics in sebaceous gland morphogenesis in mouse skin. Dev Biol 2012; 363:138-146.

27 Vidal VP, Chaboissier MC, Lutzkendorf S, et al: Sox9 is essential for outer root sheath differentiation and the formation of the hair stem cell compartment. Curr Biol 2005;15:1340-1351.

28 Nowak JA, Polak L, Pasolli HA, Fuchs E: Hair follicle stem cells are specified and function in early skin morphogenesis. Cell Stem Cell 2008;3:33-43.

29 Miyazaki M, Man WC, Ntambi JM: Targeted disruption of stearoyl-CoA desaturase1 gene in mice causes atrophy of sebaceous and meibomian glands and depletion of wax esters in the eyelid. J Nutr 2001;131:2260-2268.

30 Zheng Y, Eilertsen KJ, Ge L, et al: Scd1 is expressed in sebaceous glands and is disrupted in the asebia mouse. Nat Genet 1999;23:268-270.

31 Laederich MB, Funes-Duran M, Yen L, et al: The leucine-rich repeat protein LRIG1 is a negative regulator of ErbB family receptor tyrosine kinases. J Biol Chem 2004;279:47050-47056.

32 Herbst RS: Review of epidermal growth factor receptor biology. Int J Radiat Oncol Biol Phys 2004;59(suppl 2):21-26.

33 Suzuki Y, Miura H, Tanemura A, et al: Targeted disruption of LIG-1 gene results in psoriasiform epidermal hyperplasia. FEBS Lett 2002;521:67-71.

34 Dahlhoff M, Frances D, Kloepper JE, et al: Overexpression of epigen during embryonic development induces reversible, epidermal growth factor receptor-dependent sebaceous gland hyperplasia. Mol Cell Biol 2014;34: 3086-3095.

35 Frances D, Sharma N, Pofahl R, et al: A role for Rac1 activity in malignant progression of sebaceous skin tumors. Oncogene 2015;34:5505-5512.

36 Troy JL, Ackerman AB: Sebaceoma. A distinctive benign neoplasm of adnexal epithelium differentiating toward sebaceous cells. Am J Dermatopathol 1984;6:7-13.

37 Tanemura A, Nagasawa T, Inui S, Itami S, et al: LRIG-1 provides a novel prognostic predictor in squamous cell carcinoma of the skin: immunohistochemical analysis for 38 cases. Dermatol Surg 2005;31:423-430.

38 Nakamura T, Hamuro J, Takaishi M, et al: LRIG1 inhibits STAT3-dependent inflammation to maintain corneal homeostasis. J Clin Invest 2014;124:385-397.

39 Lindquist D, Kvarnbrink S, Henriksson R, Hedman H: LRIG and cancer prognosis. Acta Oncol (Stockholm) 2014;53:1135-1142. 
40 Powell AE, Wang Y, Li Y, et al: The pan-ErbB negative regulator Lrig1 is an intestinal stem cell marker that functions as a tumor suppressor. Cell 2012;149:146-158.

41 Krig SR, Frietze S, Simion C, et al: Lrig1 is an estrogen-regulated growth suppressor and correlates with longer relapse-free survival in ERalpha-positive breast cancer. Mol Cancer Res 2011;9:1406-1417.

42 An Y, Zhao Z, Ou P, Wang G: Expression of LRIG1 is associated with good prognosis for human non-small cell lung cancer. Medicine 2015;94:e2081.

43 Kvarnbrink S, Karlsson T, Edlund K, et al: LRIG1 is a prognostic biomarker in non-small cell lung cancer. Acta Oncol (Stockholm) 2015;54:1113-1119.

44 Thompson PA, Ljuslinder I, Tsavachidis S, et al: Loss of LRIG1 locus increases risk of early and late relapse of stage I/II breast cancer. Cancer Res 2014;74:2928-2935.

$45 \mathrm{Su} \mathrm{J}, \mathrm{Wu} \mathrm{S}, \mathrm{Wu} \mathrm{H}, \mathrm{Li} \mathrm{L}$, Guo T: CD44 is functionally crucial for driving lung cancer stem cells metastasis through Wnt/ $\beta$-catenin-FoxM1-Twist signaling. Mol Carcinog 2015, E-pub ahead of print.

46 Chanmee T, Ontong P, Kimata K, Itano N: Key roles of hyaluronan and its CD44 receptor in the stemness and survival of cancer stem cells. Front Oncol 2015;5:180.

47 Chen Y, Fu Z, Xu S, Xu Y, Xu P: The prognostic value of CD44 expression in gastric cancer: a meta-analysis. Biomed Pharmacother 2014;68:693-697.

48 Luo Z, Wu RR, Lv L, et al: Prognostic value of CD44 expression in non-small cell lung cancer: a systematic review. Int J Clin Exp Pathol 2014;7:3632-3646.

49 Montgomery E, Abraham SC, Fisher C, et al: CD44 loss in gastric stromal tumors as a prognostic marker. Am J Surg Pathol 2004;28:168-177.

50 Kim N, Choung HK, Lee MJ, Khwarg SI, Kim JE: Cancer stem cell markers in eyelid sebaceous gland carcinoma: high expression of ALDH1, CD133, and ABCG2 correlates with poor prognosis. Invest Ophthalmol Vis Sci 2015; 56:1813-1819.

51 Bhaijee F, Brown AS: Muir-Torre syndrome. Arch Pathol Lab Med 2014;138:1685-1689.

52 Peterson SC, Eberl M, Vagnozzi AN, et al: Basal cell carcinoma preferentially arises from stem cells within hair follicle and mechanosensory niches. Cell Stem Cell 2015;16:400-412.

53 Barnes et al: Senescent-atrophic epidermis retains Lrig1+ stem cells and loses Wnt signalling, a phenotype shared with the CD44KO mouse, in preparation.

54 Cottle DL, Kretzschmar K, Schweiger PJ, et al: c-MYC-induced sebaceous gland differentiation is controlled by an androgen receptor/p53 axis. Cell Rep 2013;3:427-441. 